\title{
GeroScience: understanding the interaction of processes of aging and chronic diseases
}

\author{
William E. Sonntag • Zoltan Ungvari
}

Published online: 6 December 2016

(C) American Aging Association 2016

After careful deliberation, the American Aging Association (AAA) has decided to change the title of its journal from AGE to GeroScience, effective January 2017. This title change is intended to reflect a new direction and commitment to understanding the interaction of aging processes and pathogenesis of chronic diseases.

The Association and editorial board members have made substantial commitments and efforts with the aim of making GeroScience a premier publication in this field. The dedication and vision of those involved is greatly appreciated.

GeroScience is an international, peer-reviewed journal that publishes research articles focusing on the association between aging and genesis of chronic diseases. True to the concept of Geroscience, the journal will focus on mechanistic studies using clinically relevant models of aging and chronic age-related diseases that provide novel information relevant for increasing the healthspan/quality of life and lifespan in the elderly.

GeroScience welcomes manuscripts on specific mechanisms that intersect aging and chronic disease pathways, including:

chronic low-grade inflammation,

cellular senescence,

macromolecular damage,

oxidative-nitrative stress,

W. E. Sonntag $(\bowtie) \cdot$ Z. Ungvari

University of Oklahoma Health Science Center, Oklahoma, OK, USA

e-mail: William-Sonntag@ouhsc.edu maladaptation to cellular and molecular stresses, impaired stem cell function and regeneration, alterations in proteostasis, epigenetic dysregulation, impaired mitochondrial function and cellular metabolism.

GeroScience is especially interested in manuscripts developing innovative strategies to improve cardiovascular, neurocognitive, and musculoskeletal health-span and welcomes studies using a variety of experimental approaches, including in vivo studies and investigations using isolated tissue preparations and cultured cells.

GeroScience will also publish articles seeking to understand the molecular and cellular mechanisms underlying aging processes. Studies using single cell organisms to humans are welcome. Clinical studies will be considered but the results should reveal underlying biological mechanisms of aging that impact aging and agerelated disease. These studies must address more than issues related to the care and treatment of geriatric patients or exercise regimens designed to improve function. Papers concerned with social, economic, and political issues of aging will generally not be considered unless they relate directly to biomedical gerontology.

In addition to manuscripts emerging from original research, GeroScience actively solicits and welcomes reviews on important topics in biomedical gerontology. Solely for review articles, authors should email a pre-submission inquiry to the Editor-in-Chief (william-sonntag@ouhsc.edu) or the 
Deputy Editor (zoltan-ungvari@ouhsc.edu) which includes a working title, authors and affiliations, abstract and brief outline of the content.

GeroScience strives to achieve efficient peer-review times and to be as author-friendly as possible. Articles undergo an initial review by an Associate Editor to ensure that the study is appropriate for GeroScience. The article is then assigned to external reviewers in the field.

Our vision is that the editors of GeroScience will actively assist the authors to improve their papers by selecting reviewers with complementary expertise who can help highlight the clinical relevance and translatability of preclinical findings into clinical interventions.

The first articles for the inaugural edition are currently under review and, based on our rapid review process, there is still time for submissions. Furthermore, we have recently launched several 'Call for Papers' addressing specific areas related to Geroscience and are selecting Guest Editors for the following topics:

"Epigenetics of aging"

"Where neuroscience meets geroscience: mechanisms at the interface between aging and neurodegeneration"

"The GH/IGF-1 axis in geroscience: from regulation of cellular aging processes to the pathogenesis of age-related diseases"

"Gait and balance dysfunction in aging: from experimental models to clinical consequences"

"Neuropeptides and peptide hormones in aging"

Please use the website for submissions (https://www. editorialmanager.com/jaaa/). A detailed description of the Call-for-Papers can be accessed from www. springer.com/11357.

We look forward to your contributions! 